\title{
Effect of substrate stiffness on differentiation of umbilical cord stem cells
}

\author{
Małgorzata Witkowska-Zimny' ${ }^{1}$ Katarzyna Walenko 1,2, Anna Ewa Wałkiewicz³, Zygmunt \\ Pojda ${ }^{4}$, Jacek Przybylski¹ and Małgorzata Lewandowska-Szumieł1,2
}

'Department of Biophysics and Human Physiology, ${ }^{2}$ Department of Histology and Embryology, Centre of Biostructure Research, Medical University of Warsaw, Warszawa, Poland; ${ }^{3}$ nstitute of Physics, Polish Academy of Sciences, Warszawa, Poland; ${ }^{4}$ Department of Regenerative Medicine, WIHE Institute of Hygiene and Epidemiology

Tissue formation and maintenance is regulated by various factors, including biological, physiological and physical signals transmitted between cells as well as originating from cell-substrate interactions. In our study, the osteogenic potential of mesenchymal stromal/stem cells isolated from umbilical cord Wharton's jelly (UC-MSCs) was investigated in relation to the substrate rigidity on polyacrylamide hydrogel (PAAM). Osteogenic differentiation of UC-MSCs was enhanced on stiff substrate compared to soft substrates, illustrating that the mechanical environment can play a role in differentiation of this type of cells. These results show that substrate stiffness can regulate UC-MSCs differentiation, and hence may have significant implications for design of biomaterials with appropriate mechanical properties for regenerative medicine.

Key words: osteogenic differentiation, substrate stiffness, umbilical cord mesenchymal stromal/stem cells

Received: 17 October, 2011; revised: 03 January, 2012; accepted: 04 April, 2012; available on-line: 11 May, 2012

\section{INTRODUCTION}

Stem cell biology has become an interesting topic, especially in the context of opportunities provided by transplantation medicine for treating diseases and injuries. Mesenchymal stem cells from perinatal tissues can be particularly useful in clinical settings for autologous transplantation for fetuses and newborns, and after banking also in later stages of life, as well as for in utero transplantation in case of genetic disorders. Wharton's jelly obtained from the umbilical cord is a rich reservoir of mesenchymal stem cells. It has been shown that UC-MSCs can differentiate into several lineages, including adipose cells, chondrocytes, osteoblasts, neuronal cells, endothelial cells, cardiomyocytes, hepatocyte-like and pancreas beta cells (Wang et al., 2004; Anzalone et al., 2010; 2011). In this context, UC-MSCs are an example of multipotent or even pluripotent stem cells. In the last few years, MSCs derived from Wharton's jelly have been of particular interest because of their advantages over cells of embryonic and somatic origin. Firstly, they are derived from an ethically uncontroversial and practically inexhaustible source, and may be harvested noninvasively at low cost. Secondly, UC-MSCs do not induce teratomas - on the contrary, they have anticancer effects (Witkowska-Zimny \& Wrobel, 2011). Therefore, UC-MSCs are attractive cells for various medical application.
Tissue engineering is a new promising tool in reconstructive surgery. Restoration of bone function by utilizing tissue-engineering technologies often requires the use of a scaffold in which cells can adhere, grow and proliferate in order to regenerate the damaged tissue. There are many important aspects of the scaffold parameters (i.e., chemical composition, surface topography, scaffold porosity, pore size, interconnectivity, and mechanical properties) that can influence cell growth, differentiation and behavior ( $\mathrm{Kim}$ et al., 2010). For tissue engineering applications, it is crucial to understand the processes of tissue formation and regeneration which will point us towards the creation of a new functional tissue. Tissues are not equally flexible, e.g., brain tissue is considered to be soft $(\sim 1 \mathrm{kPa})$ whilst bone tissue is hard $(\sim 100 \mathrm{kPa})$. During embryogenesis cells are exposed not only to chemical signals but also to physical forces influencing their differentiation. In 2006 intriguing data published by Engler et al. showed the significance of matrix support stiffness for stem cell differentiation. Since it could have practical implications for tissue engineering, in this work we analyzed the response of human mesenchymal stem cells isolated from umbilical cord Wharton's jelly toward substrate elasticity with particular attention paid to osteogenic signal expression and differentiation. Due to the UC-MSCs ability to differentiate into multiple cell types, it is important to optimize the chemical, biological and physical stimuli that may induce osteogenic differentiation of these cells in contact with the substrate/scaffold. The objective of this study was to evaluate if substrate stiffness affects the in vitro response of UC-MSCs to osteogenic stimuli. To the best of our knowledge, this is the first report on the impact of substrate stiffness on the behavior of perinatal stem cells.

\section{MATERIALS AND METHODS}

Cell culture. Low passage number human UC-MSCs obtained from the Institute of Oncology in Warsaw (isolated from umbilical cords, according to methods described by McElreavey et al. (1991) were cultured in osteogenic medium under standard conditions $\left(37^{\circ} \mathrm{C}\right.$, $\left.5 \% \mathrm{CO}_{2}\right)$ for 14 days. Osteogenic differentiation was induced using Dulbecco modified Eagle medium (DMEM; Gibco BRL) supplemented with 20\% fetal bovine serum

e-mail: mwitkowska@wum.edu.p

Abbreviations: AFM, atomic force microscopy; BM-MSCs, bone marrow mesenchymal stem cells; GS, glass coverslips; MSCs, mesenchymal stem cells; PAAM, polyacrylamide hydrogel; UC-MSCs, umbilical cord mesenchymal stromal/stem cells. 
(FBS), $100 \mathrm{nM}$ dexamethasone, $50 \mu \mathrm{M}$ ascorbic acid and $10 \mathrm{mM} \beta$-glycerophosphate. Cells were plated at a density of $1 \times 10^{5} \mathrm{cells} / \mathrm{cm}^{2}$. Media changes were performed every 4 days. All cell culture reagents and chemicals were obtained from Invitrogen and Sigma-Aldrich, unless stated otherwise. The study was performed according to the recommendation of the 2 nd Local Ethical Committee at the Medical University of Warsaw.

Preparation of substrates characterized by different stiffness. Polyacryalmide (PAAM) substrates were prepared according to Wang and Pelham protocol (1998). Round glass coverslips were treated with 3 -aminopropyltrimethoxysilane and $0.5 \%$ glutaraldehyde. PAAM gel solution with a desired concentration of acrylamide and bis-acrylamide (Bio-Rad) was allowed to polymerize to form a layer of gel on the slides. The flexibility of the substrate was manipulated by varying the acrylamide and bis-acrylamide ratio. After being exposed to UV light for $10 \mathrm{~min}$ two times in a row, the polyacrylamide sheet was washed twice and incubated with a solution of type I collagen $(0.2 \mathrm{mg} / \mathrm{ml})$ overnight at $4^{\circ} \mathrm{C}$. Sulfo-SANPAH (Pierce) was used to link collagen-I to the PAAM gel surface. Stiff substrates (glass coverslips) were coated with the same density of collagen-I. Before cells were plated, the substrates were soaked in PBS and then in DMEM at room temperature.

Characterization of gel substrates. The microelasticity of PAAM gels was determined using a atomic force microscopy PicoPlus 5500 Agilent AFM. Indentation of the studied gels was carried out in a liquid (PBS buffer, $\mathrm{pH} 7.4$ ), at $21^{\circ} \mathrm{C}$. Before indentation, the system was left for $2 \mathrm{~h}$ to equilibrate. Five different areas of each gel were chosen to be indented and each area was subjected to 10 indentation cycles. All gels were probed with the same AFM tip (Veeco, DNPS) of the nominal spring constant $k=0.06 \mathrm{Nm}^{-1}$ and speed set to $10 \mathrm{~ms}^{-1}$. Young module (E) was calculated from the collected force - indentation curves by fitting to the classical Hertz model for cone-shaped geometry, according to the procedure described by Engler and Mahaffy (Engler et al., 2004; Mahaffy et al., 2004). For the calculations, the value of 0.5 was taken as the Poison ratio and the cone angle was set at $\alpha_{1 / 2}=12^{\circ}$.

Real time PCR. Total RNA was extracted from cells using RNeasy kit (Qiagen) according to the manufacturer's protocol and RNA concentration was assessed using a Nanodrop spectrofotometr (Thermo Scientific). cDNA was subsequently prepared from $250 \mathrm{ng}$ of RNA template by reverse transcription kit (Applied Biosystems). The expression of Runx2, osteocalcin (OC) and collagen type I after 14 days of culture on three different substrates was subsequently determined by real time PCR (Assays IDs: Hs00231692_m1, Hs01587814_g1, Hs00164004_m1, Applied Biosystems) using a 7500Fast Real Time PCR System (Applied Biosystems). Each sample was analyzed in triplicate. The relative expression of the target genes was normalized to the reference gene GAPDH (Hs99999905_m1) and the data was analyzed using the $\Delta \Delta \mathrm{Ct}$ method.

Alizarin Red S staining. Cells were cultured on stiff or soft gel-coated slides and at day 14 they were fixed with 4\% paraformaldehyde and washed twice in PBS. Next, $0.5 \mathrm{ml} \mathrm{1 \%}$ Alizarin Red S, pH 4.2 was added to each well for 10 minutes. Cultures were washed in distilled water until no further red stain was released from the cell layers, and then were left to air-dry. Cultures were visualized using an inverted microscope (Nikon, Eclipse TE 2000-U). Each culture was performed in du- plicate and the experiments were repeated at least two times.

Statistical analysis. Statistical analysis of the results was carried out by nonparametric ANOVA (Kruskal Willis test with Dunn's post hoc test). Differences at the level of $p<0.05$ were accepted as significant. The analysis was performed using GraphPad Pism software.

\section{RESULTS}

The mechanical properties of polyacrylamide can be easily modified by altering the concentration of crosslinks in the gel. Increasing the concentration of acrylamide or bis-acrylamide monomer results in a higher stiffness of the gel after polymerization which is cor-

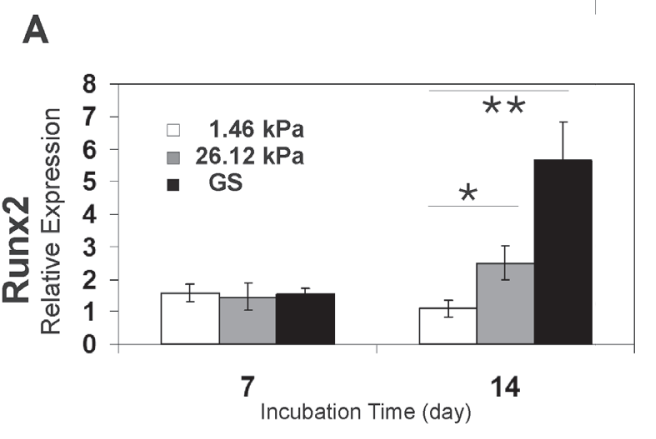

B
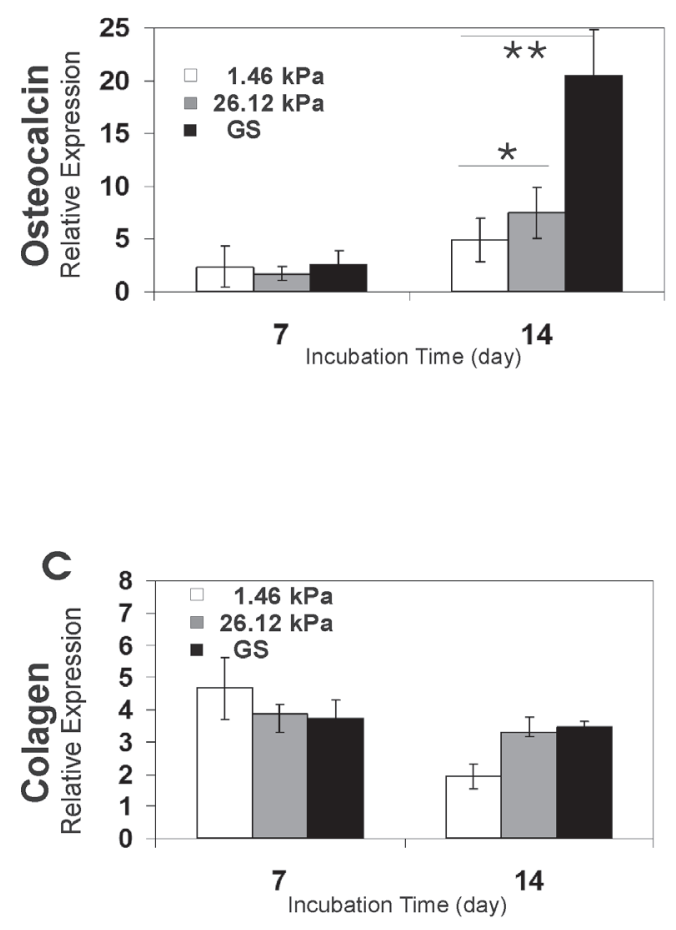

Figure 1. Gene expression levels of osteogenic markers on substrates of different stiffness.

Relative expression of Runx2 (A) and osteocalcin (B) is higher in UC-MSCs grown on more rigid substrate as compared to the softer one. Statistical significance of the differences between the results are indicated $\left({ }^{*} p<0.05 ;{ }^{* *} p<0.01\right)$. No statistically significant differences were observed between groups for collagen I (C). The gene expression was normalised to the reference gene, GAPDH. 

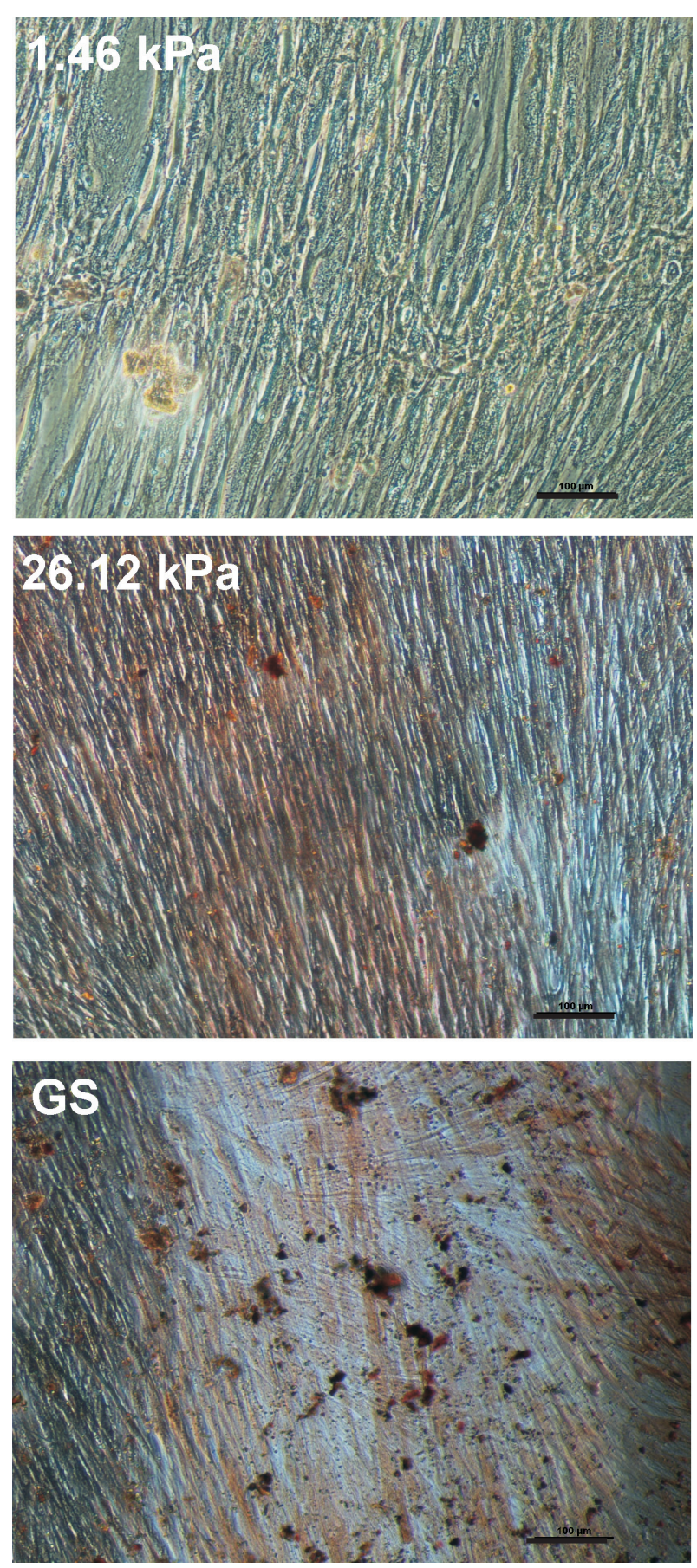

Figure 2. Assessment of mineralization - visualization of calcium deposition on day 14 .

Calcium deposition positively correlates with substrate stiffness. Scale bars represent $100 \mu \mathrm{m}$.

related with the higher Young's modulus. In this way gels with two different stiffness values were obtained: $1.46 \mathrm{kPa}$ and $26.12 \mathrm{kPa}$; and glass coverslips (GS) devoid of a PAAM layer served as a "high rigidity" control. We cultured UC-MSCs on flexible collagen-coated polyacrylamide substrates with similar chemical properties but different rigidity, and differentiation of UC-MSCs toward osteogenic precursors was determined. The coverage of collagen on the substrates allows correct cell adhesion. Cell viability (determined by the X'T'T assay) and morphology were comparable on all substrates used (data not shown). The process of osteogenesis can be characterized by well-defined bone formation markers as: alkaline phosphatase, collagen type I, osteocalcin and Runx2. In our study, differentiation was verified by: (i) demonstrating the induction of expression of osteoblastspecific indicator genes: Runx 2 - key transcription factor of osteoblastogenesis, and osteocalcin - a marker of osteogenesis and mineralization; (ii) calcium deposit. Runx 2 and the osteocalcin gene expression were higher on the more rigid substrate than on the softer one. Collagen type I as an early stage bone formation marker, showed no significant differences in expression level over time on all substrates used (Fig. 1). The level of expression of osteonectin (secreted glycoprotein synthesized constitutively by osteoblasts) in all cultured UC-MSCs remained constant (data not shown). Mineral staining was performed after 14 days of growth in osteogenic medium by using Alizarin S Red. The assessment of mineralization - visualization of calcium deposition was also positively correlated with the substrate stiffness (Fig. 2).

\section{DISCUSSION}

In the present work we focused on UC-MSCs as a therapeutic cell source that can be induced to differentiate into on osteogenic cell lineage. The results demonstrate that the UC-MSCs differentiation into osteoblasts varies depending on the substrate stiffness. Cell attachment and adhesion do not differ between the substrates used. Several studies have shown that adhesion and subsequent BM-MSCs attachment vary with substrate stiffness (Sharma \& Snedeker, 2010; Park et al., 2011). We did not observe a similar effect for UC-MSCs, which indicates that the substrate stiffness affects the UC-MSCs osteogenic differentiation through mechanisms other than cell attachment and adhesion. The differences between the present and ealier results may be related to the different origins of the stem cells used. UC-MSCs differ from BM-MSCs by, e.g., upregulation of extracellular metalloproteinases MMP-1 and MMP-2 and downregulation of osteogenic markers osteopontin and alkaline phosphatase on matrix under osteogenic conditions (Hou et al., 2009). We also do not observe expression of osteopontin or alkaline phosphatase during the UC-MSCs osteogenic differentiation (data not shown). However, the observed differences did not affect the final result of osteogenic differentiation of these perinatal umbilical mesenchymal stem cells. The studies of Schneider et al. (2010) also suggest different mechanisms of UC-MSCs for bone cell formation compared to BM-MSCs. The osteogenic differentiation properties of UC-MSCs are enhanced by the contact with collagen, whereas the contact between BM-MSCs stimulates the synthesis of extracellular matrix proteins, including the collagen type I, III and IV.

Several authors have reported that cell behavior (proliferation and differentiation) is modulated by substrate rigidity to a degree dependent on the substrate stiffness in relation to the stiffness of the native tissue (Pelham et al., 1997). Bone tissue is rigid, so it could be assumed that a substrate of greater stiffness would favor osteogenic differentiation more than a substrate of lower stiffness. Here, UC-MSCs grown on more rigid surfaces exhibited characteristics corresponding to more mature osteoblasts as determined by measuring expression of mRNA for the most important osteogenic lineage-specific factors Runx2 and osteocalcin. The highest expression was observed on glass slides. These findings are 
in agreement with the studies by Evans and coworkers (2009) who demonstrated that expression of osteogenic regulatory genes, e.g. Runx2 by embryonic stem cells, was the highest on plain tissue culture plates than on softer gels. In our studies we did not see high expression of collagen type I, a component of bone extracellular matrix. This finding is consistent with previous studies for osteogenic potential of pre-osteoblast cells on collagen scaffolds (Keogh et al., 2010). This could be related to the coverage of the substrates with collagen providing sufficient extracellular amount of this protein and thereby blocking its endogenous expression.

The strong osteogenic response of the UC-MSCs on the more rigid substrate, also confirmed by mineralization staining is attributed to the fact that such a substrate best mimics the natural bone microenvironment. The favorable biological features and the absence of ethical issues concerning the application of UC-MSCs suggest that these cells could be promising candidates for tissue engineering and stem cell therapy. The understanding of the mechanisms and molecular processes underlying stem cell differentiation and cell fate determination is not complete. Here we showed that the stiffness of the cell adhesion substrates modulates differentiation of these perinatal stem cells. The role of integrins as signaling molecules responsible for cellular adhesion and probably mechanosensitivity will be a subject of our future investigations. We find interesting the influence substrate rigidity on cells destiny since tumor cells lost the ability to respond to such stimuli. Further studies will elucidate the potential for the regulation of cell behavior by mechanical signals. A better understanding of these interactions can have implications for developing cell-based therapies to promote bone formation and osseointegration.

\section{Acknowledgements}

This work was supported by grant No. N N302157037 from the National Center of Science. We thank the Laboratory of Biological Physics SL-4 of the Institute of Physics PAS for the access to the AFM equipment.

\section{REFERENCES}

Anzalone R, Lo Iacono M, Corrao S, Magno F, Loria T, Cappello F, Zummo G, Farina F, La Rocca G (2010) New emerging potentials for human Wharton's jelly mesenchymal stem cells: immunological features and hepatocyte-like differentiative capacity. Stem Cells Dev 19: 423-438.

Anzalone R, Lo Iacono M, Loria T, Di Stefano A, Giannuzzi P, Farina F, La Rocca G (2011) Wharton's jelly mesenchymal stem cells as candidates for beta cells regeneration: extending the differentiative and immunomodulatory benefits of adult mesenchymal stem cells for the treatment of type 1 diabetes. Stem Cell Rev 7: 342-363.

Engler AJ, Richert L, Wong JY, Picart C, Discher DE (2004) Surface probe measurements of the elasticity of sectioned tissue, thin gels and polyelec- trolyte multilayer films: correlations between substrate stiffness and cell adhesion. Surf Sci 570: 142-154.

Engler AJ, Sen S, Sweeney HL, Discher DE (2006) Matrix elasticity directs stem cell lineage specification. Cell 126: 677-689.

Evans ND, Minelli C, Gentleman E, LaPointe V, Patankar SN, Kallivretaki M, Chen X, Roberts CJ, Stevens MM (2009) Substrate stiffness affects early differentiation events in embryonic stem cells. Eur Cell Mater 18: 1-14.

Hou T, Xu J, Wu X, Xie Z, Luo F, Zhang Z, Zeng L (2009) Umbilical cord Wharton's Jelly: a new potential cell source of mesenchymal stromal cells for bone tissue engineering. Tissue Eng Part A 15: 2325-2334.

Keogh MB, O'Brien FJ, Daly JS (2010) Substrate stiffness and contractile behaviour modulate the functional maturation of osteoblasts on a collagen-GAG scaffold. Acta Biomater 6: 4305-4313.

Kim K, Yeatts A, Dean D, Fisher, JP (2010) Stereolithographic bone scaffold design parameters: osteogenic differentiation and signal expression. Tissue Eng Part B Rev 16: 523-539.

Mahaffy RE, Park S, Gerde E, Kas J, Shih CK (2004) Quantitative analysis of the viscoelastic properties of thin regions of fibroblasts using atomic force microscopy. Biophys J 86: 1777-1793.

McElreavey KD, Irvine AI, Ennis KT, McLean WH (1991) Isolation, culture and characterisation of fibroblast-like cells derived from the Wharton's jelly portion of human umbilical cord. Biochem Soc Trans 19: $29 \mathrm{~S}$.

Park JS, Chu JS, Tsou AD, Diop R, Tang Z, Wang A, Li S (2011) The effect of matrix stiffness on the differentiation of mesenchymal stem cells in response to TGF-beta. Biomaterials 32: 3921-3930.

Pelham RJ, Jr, Wang Y (1997) Cell locomotion and focal adhesions are regulated by substrate flexibility. Proc Natl Acad Sci USA 94: 13661-13665.

Schneider RK, Puellen A, Kramann R, Raupach K, Bornemann J, Knuechel R, Perez-Bouza A, Neuss S (2010) The osteogenic differentiation of adult bone marrow and perinatal umbilical mesenchymal stem cells and matrix remodelling in three-dimensional collagen scaffolds. Biomaterials 31: 467-480.

Sharma RI, Snedeker J G (2010) Biochemical and biomechanical gradients for directed bone marrow stromal cell differentiation toward tendon and bone. Biomaterials 31: 7695-7704.

Wang HS, Hung SC, Peng, ST, Huang CC, Wei HM, Guo YJ, Fu YS, Lai MC, Chen CC (2004) Mesenchymal stem cells in the Wharton's jelly of the human umbilical cord. Stem Cells 22: 1330-1337.

Wang YL, Pelham RJ, Jr (1998) Preparation of a flexible, porous polyacrylamide substrate for mechanical studies of cultured cells. Methods Ensymol 298: 489-496.

Witkowska-Zimny M, Wrobel E (2011) Perinatal sources of mesenchymal stem cells: Wharton's jelly, amnion and chorion. Cell Mol Biol Lett 16: 493-514. 